\title{
Geschlecht in universitären Kontexten: eine qualitative Studie über Wissenschaftlerinnen in Österreich
}

\author{
Nina-Sophie Fritsch* \\ * Institut für Soziologie, Universität Wien, AT-1090 Wien, nina.fritsch@univie.ac.at.
}

\section{Abstracts \\ Geschlecht in universitären Kontexten: eine qualitative Studie über Wissenschaftlerinnen in Österreich}

Die universitäre Wissenschaft ist in Österreich durch eine vertikale Geschlechtersegregation geprägt. Bei höheren Berufspositionen steht ein Männeranteil von 80 Prozent einem Frauenanteil von 20 Prozent gegenüber. Dieser Beitrag widmet sich dieser Unterrepräsentanz und untersucht Barrieren für Wissenschaftlerinnen in unterschiedlichen organisationalen Kontexten. Als empirische Grundlage dienen 22 problemzentrierte Interviews. Die Analyse des Materials zeigt, dass die Themen soziale Netzwerke, geschlechtsspezifische Praktiken im universitären Arbeitsalltag und die Vereinbarkeitsproblematik kritische Bereiche in den Lebensentwürfen der Wissenschaftlerinnen darstellen. Die vorliegende Studie verdeutlicht jedoch auch, dass differierende organisationale Kontexte eine bedeutsame Rolle spielen und dabei nicht ausschliesslich negative Folgen für die Wissenschaftlerinnen produzieren.

Schlüsselwörter: Wissenschaftliche Berufsbiografien, Barrieren für Frauen, organisationale Kontexte, geschlechtsspezifische Praktiken, Österreich

\section{Les femmes dans les contextes académiques: une étude qualitative sur les femmes de science en Autriche}

En Autriche, la communauté scientifique est marquée par une ségrégation verticale des sexes. Aux niveaux supérieurs, la part des hommes de science se chiffre à 80 pour cent tandis que les femmes n'occupent que 20 pour cent des postes scientifiques. Le présent article porte sur la sous-représentation féminine et examine les obstacles auxquels les femmes de science doivent faire face dans divers contextes organisationnels. La base empirique comprend 22 interviews centrées sur les problèmes rencontrés. Les données recueillies indiquent que les réseaux sociaux, les pratiques professionnelles quotidiennes spécifiques au genre, de même que la problématique de la conciliation travail-famille constituent pour les femmes de science des barrières dans la réalisation de leur projet de vie. Toutefois, la présente étude souligne aussi que les différents contextes organisationnels jouent un rôle important et n'entraînent pas seulement des conséquences négatives pour les chercheuses.

Mots-clés: Parcours professionnel académique, obstacles rencontrés par les femmes, contextes organisationnels, pratiques spécifiques au genre, Autriche

\section{Gender in Academic Contexts: A Qualitative Study on Female Scientists in Austria}

Academic science in Austria is characterized by vertical gender segregation. Higher levels of professional positions show a share of $80 \%$ of male compared to $20 \%$ of female academics. This article deals with the female underrepresentation in academia and analyses barriers and their consequences in different organizational contexts. The findings are based on 22 problem-centered interviews. The results emphasize social networks, gendered practices in everyday work and the work-life balance as crucial barriers for women. Nevertheless, the analysis also shows that different organizational contexts play an important role and do not only produce negative consequences for female academics.

Keywords: Academic occupational careers, obstacles for women, organizational contexts, gendered practices, Austria 


\title{
Geschlecht in universitären Kontexten: eine qualitative Studie über Wissenschaftlerinnen in Österreich ${ }^{1}$
}

\author{
Nina-Sophie Fritsch*
}

\section{$1 \quad$ Einleitung}

Wissenschaftliche Karrierewege von Männern und Frauen unterscheiden sich deutlich voneinander. Dabei ist das Ungleichverhältnis zwischen den Geschlechtern in der universitären Wissenschaft keine Besonderheit einzelner Länder, sondern ein globales Phänomen (Benschop und Bourns 2003; Blickenstaff 2005; Lind 2007; European Commission 2013). Mit wenigen Ausnahmen ist das Geschlechterverhältnis zu Ungunsten von Wissenschaftlerinnen verteilt. Obwohl politische Initiativen zur Verbesserung der Chancengleichheit in den letzten Jahren zunehmend eingesetzt werden, bleiben vor allem die oberen Positionsstufen der wissenschaftlichen Karriereleiter grossteils männlich dominiert. Dies lässt darauf schliessen, dass die Barrieren, auf die Wissenschaftlerinnen trotz aller Reformbemühungen stossen, tief im Wissenschaftssystem verankert sind (Bielby 2000, 69).

Der vorliegende Beitrag widmet sich dieser Unterrepräsentanz. Im Zentrum des Artikels stehen wissenschaftliche Berufsbiografien von Frauen in Österreich. Der Schwerpunkt der Analysen liegt auf den Barrieren für Wissenschaftlerinnen und deren Folgen. Die nachstehenden Fragestellungen sind dabei forschungsleitend: Mit welchen Barrieren sind Frauen in ihrer wissenschaftlichen Berufsbiografie an österreichischen Universitäten konfrontiert? Und welche Folgen resultieren aus unterschiedlichen organisationalen Kontexten? Als Datenbasis dienen 22 qualitative Interviews. Dabei setzt sich das Interviewmaterial aus zwei Fallgruppen zusammen. Es werden Interviews mit Frauen integriert, die aufgrund von Barrieren auf unterschiedlichen Positionen aus dem universitären System ausgestiegen sind. ${ }^{2}$ Zusätzlich werden Wissenschaftlerinnen zum Thema befragt, die trotz dieser Barrieren eine langfristige und erfolgreiche Karriere vorweisen. Der systematische Vergleich hebt Unterschiede

\footnotetext{
* Institut für Soziologie, Universität Wien, AT-1090 Wien, nina.fritsch@univie.ac.at.

1 Die Autorin bedankt sich bei Raimund Haindorfer, Cornelia Schadler, Roland Verwiebe und Ulrike Zartler für die hilfreiche und wertvolle Unterstützung bei der Bearbeitung dieses Forschungsprojekts.

2 Ein Ausstieg aus dem universitären Wissenschaftssystem wird durch institutionelle Bedingungen und individuelles Handeln bestimmt und ist Teil eines Berufsverlaufs. In Anlehnung an sozialwissenschaftliche Studien in diesem Bereich (Wolfinger et al. 2008, 389) wird der berufliche Verlauf als «Pipeline-Modell» verstanden, bei dem die Anzahl der zur Verfügung stehenden Positionen mit aufsteigender hierarchischer Stufe abnimmt.
} 
und Gemeinsamkeiten in den Berufsbiografien der Wissenschaftlerinnen hervor, die entlang der Themenbereiche (1) soziale Netzwerke, (2) Praktiken im universitären Arbeitsalltag und (3) Vereinbarkeit von beruflichen und privaten Verpflichtungen präsentiert werden. Die Relevanz der vorliegenden Untersuchung liegt in der Stärkung des Wissens um Barrieren für wissenschaftliche Karrieren von Frauen und bildet daher eine Ergänzung und Erweiterung zu bisherigen Studien (Raddon 2002; Lind 2007; Elg und Jonnergard 2010; Kahlert 2011; Kahlert 2013).

\section{Forschungsdesiderat: die Situation von Wissenschaftlerinnen in der universitären Wissenschaft}

Wissenschaftliche Karriereverläufe von Frauen sind im Allgemeinen durch vertikale Segregation geprägt (Lind 2007). Europaweit sind Wissenschaftlerinnen auf den höheren akademischen Positionen an universitären Einrichtungen unterrepräsentiert. Nur ca. 10 Prozent aller EU-27 Universitäten werden von Frauen geführt (European Commission 2013). Der deutschsprachige Hochschulraum - mit Österreich, Deutschland und der Schweiz - weist ähnliche Geschlechterverhältnisse vor: Während in Deutschland der Anteil von Frauen in den obersten Positionen bei nur ca. 15 Prozent liegt, liegen die Werte in der Schweiz mit 26 Prozent etwas höher (European Commission 2013).

Das ungleiche Geschlechterverhältnis an österreichischen Universitäten ist in Abbildung 1 dargelegt: Obwohl etwas mehr als die Hälfte der Studierenden weiblich ist und auch mehr Frauen als Männer erste Abschlüsse ablegen, geht der Frauenanteil bei höheren Abschlüssen deutlich zurück. Diese Tendenz verstärkt sich in den wissenschaftlichen Berufspositionen. Frauen sind bei Universitätsassistenzstellen und als Dozentinnen deutlich unterrepräsentiert. Am oberen Ende der Karriereleiter steht ein Männeranteil von fast 80 Prozent einem Frauenanteil von 20 Prozent gegenüber. ${ }^{3}$

Die quantitativen Dimensionen der Ungleichverhältnisse sind umfassend dokumentiert (European Commission 2013; uni:data 2014) und inzwischen Gegenstand zahlreicher Reformbemühungen und Frauenförderungsprogrammen. ${ }^{4}$ Diese Gleichstellungsprogramme wurden in den letzten Jahren auf unterschiedlichen politischen Ebenen und Bereichen des gesellschaftlichen Zusammenlebens implementiert. Neben nationalstaatlich-individuellen Programmen, gibt es eine Reihe von supranationalen Massnahmen, die das Ziel der Gendergleichheit haben. Bemerkenswert ist allerdings auch, dass man trotz des Einsatzes dieser - in unterschiedlichem Ausmass erfolgrei-

3 Deutliche Unterschiede sind auch nach Wissenschaftsdisziplin zu beobachten: Besonders stark ausgeprägt ist das Ungleichgewicht zwischen Männern und Frauen in den technischen und naturwissenschaftlichen Disziplinen (uni:data 2014).

4 Ein Beispiel für eine nationale Initiative zur Förderung von Frauen in der Wissenschaft ist das Programm «Excellentia» in Österreich (Wroblewski und Leitner 2011). 
Abbildung 1 Frauen-/Männeranteil unter Studierenden und wissenschaftlichem Personal an österreichischen Universitäten (2012)

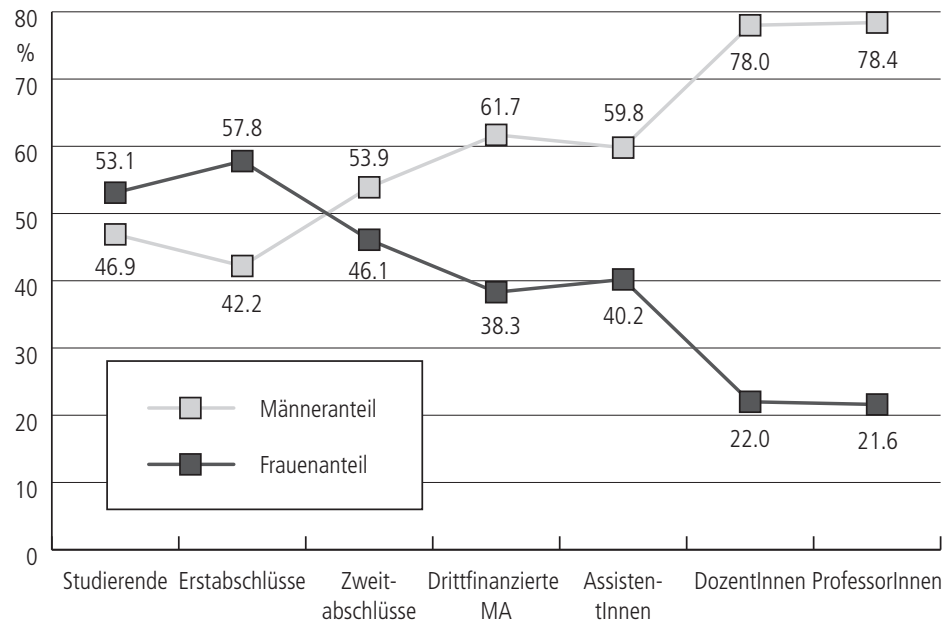

Quelle: uni:data (2014); eigene Berechnungen.

chen - Programme von der vollständigen Gleichstellung zwischen Männern und Frauen vor allem auf den höheren Berufspositionen immer noch weit entfernt ist. Dies wirft daher die Frage auf, ob es verstärkt um die Veränderung der weiblichen Beschäftigungsbedingungen innerhalb der Organisationen gehen sollte, um die Situation für Wissenschaftlerinnen nachhaltig und dauerhaft zu verbessern. Dazu ist eine systematische Analyse der Bedingungen für Frauen in den Organisationen und deren Kontexten notwendig. Dieses Forschungsdesiderat nimmt der vorliegende Beitrag auf. Es geht um den Zusammenhang von Geschlecht und Organisation konkret von Geschlecht und universitärer Wissenschaft - um die Frage, welche Rolle Geschlecht in Organisationen spielt und in welchen Kontexten Prozesse der Geschlechterdifferenzierung mit welchen Folgen für Frauen einhergehen (Riegraf 2013).

\section{Erklärungsmodelle aus empirischen Studien zu Barrieren für Wissenschaftlerinnen}

In Bezug zu den strukturellen Barrieren für Frauen in der universitären Wissenschaft, heben sozialwissenschaftliche Studien, folgende Aspekte hervor. Die Teilhabe und der Zugang zu sozialen Netzwerken sind für den Fortschritt einer wissenschaftlichen 
Karriere zentral (Gersik et al. 2000; Brink und Benschop 2012). Durch die starke Internationalisierung des Hochschulraums gewinnen Netzwerke zunehmend an Bedeutung - z. B. im Bereich internationaler Forschungsförderungsprogramme (Graf et al. 2013). DeWelde und Laursen (2011) veranschaulichen die nachteiligen Konsequenzen für Frauen durch informelle Strukturen. Um dieser Barriere entgegenzuwirken, betonen andere Studien die Relevanz von Mentoring-Programmen (McGuire und Reger 2003). Dazu belegen Gardina und Kollegen (2007) für den australischen Hochschulraum, dass Nachwuchswissenschaftlerinnen in MentoringProgrammen tendenziell länger im universitären Bereich verbleiben und insgesamt erfolgreicher sind.

Neben instabilen Beschäftigungsverhältnissen werden geschlechtsspezifische Praktiken im universitären Arbeitsalltag als Faktoren beschrieben, die sich nachteilig auf weibliche Karrieren auswirken und einen potenziellen Ausstieg aus der Wissenschaft bedingen können (Benschop und Bourns 2003; Dörre und Neis 2008). Dabei spielen insbesondere geschlechtsspezifische Rekrutierungsprozesse und Routinen des Förderns eine zentrale Rolle. Mit dem Begriff der homosozialen Reproduktion (Kanter 1977) wird in der Literatur die Tendenz beschrieben, unbewusst jene Personen zu fördern, die der eigenen Person ähneln. Da vor allem die höheren Positionen immer noch grossteils männlich besetzt sind, ist von einer Verschiebung der Geschlechterverhältnisse auszugehen (Hördt 2006). Zunehmend stärker wird auch ein Arbeitszuwachs thematisiert, der durch eine fehlende Grundfinanzierung und damit einhergehende Zunahme von Drittmittelprojekten hervorgerufen wird und wissenschaftliche Karrieren erschwert (Dautzenberg 2013; Graf et al. 2013). Dabei nehmen Erwartungen über das Arbeitspensum, über die Verfügbarkeit und die zu erbringende Leistung tendenziell zu.

Andere Studien konzentrieren sich auf die Doppelbelastung durch berufliche und private Verpflichtungen (Raddon 2002; Acker und Armenti 2004; Rafnsdottir und Heijstra 2013). ${ }^{5}$ Betont wird dabei, dass wichtige Qualifikationsstufen (Promotion oder Habilitation) in die Familiengründungsphase fallen und damit den Karrierefortschritt für Frauen erschweren (Krimmer et al. 2003). Vor diesem Hintergrund ist es naheliegend, dass Wissenschaftlerinnen eher vor die Wahl «Beruf vs. Privat» gestellt werden. Während einerseits der Ausstieg aus dem Universitätssystem einen möglichen Lösungsweg darstellt, scheint andererseits Kinderlosigkeit eine weitere Alternative (Kahlert 2013). Untersuchungen zeigen, dass überdurchschnittlich viele Professorinnen kinderlos bleiben, wobei Professoren mehrheitlich Kinder haben, deren Betreuung hauptsächlich die Partnerin übernimmt (Fieder et al. 2005). ${ }^{6}$ Letztlich erschweren auch hier die unsicheren Arbeitsbedingungen bei

5 Partnerschaftliche Arrangements in Doppelkarrierepaaren gewinnen zunehmend an Relevanz. Studien zeigen, dass tendenziell Frauen in Doppelkarrierepaaren leben und sich Partner suchen, die ihre wissenschaftlichen Karrierebestrebungen unterstützen (Kahlert 2013, 148). derzahl von Akademikerinnen hervor (Buber et al. 2011). Der Vergleich zeigt: Während die 
gleichzeitig zunehmendem Druck zur geografischen Mobilität die Vereinbarkeit zusätzlich (Dörre und Neis 2008; Becker et al. 2011).

\section{Theoretische Bezüge zu soziologischen Paradigmen und zur Geschlechterforschung in der Organisationssoziologie}

Während in der Geschlechterforschung (und vor allem in der feministischen Forschung) oft die Kritik an bestehenden Herrschaftsverhältnissen im Zentrum steht und sie sich somit einer ungleichheitstheoretischen Perspektive bedient, können empirisch beobachtbare Fortschritte - also zunehmend mehr Frauen in fast allen Teilbereichen des gesellschaftlichen Zusammenlebens - in eine modernisierungstheoretische Perspektive eingebettet werden. Die dahinter liegenden Leitfragen lauten (Aulenbacher 2010): In welcher Weise verändern sich durch den gesellschaftlichen Wandel auch Ungleichheiten nach Geschlecht? In welchen Bereichen sind geschlechtlich geprägte Ungleichheiten persistent; in welchen ist eine Auflösung zu beobachten? Und gibt es in modernen Gesellschaften organisationale Kontexte, in denen Geschlechterdifferenzen stärker eingeschrieben sind als in anderen?

Der weitere theoretische Bezugsrahmen des vorliegenden Beitrags liegt somit an der Schnittstelle zwischen Modernisierungstheorie und Ungleichheitstheorie. Die modernisierungstheoretischen Ansätze in der Tradition der funktionalistischen bzw. strukturfunktionalistischen Argumentation (Durkheim 1988; Parson 1975) gehen davon aus, dass in modernen Gesellschaften frühere Differenzierungsformen - die segmentäre und stratifikatorische Differenzierung - sukzessive an Bedeutung verlieren, was zu einer stärkeren Inklusion aller Menschen (unabhängig vom Geschlecht) in unterschiedliche Funktionsbereiche führt (Heintz und Nadai 1998, 77). Diese Argumentation negiert zwar (empirisch beobachtbare) geschlechtsspezifische Ungleichheit nicht, räumt diesen Ungleichheiten aber auch keinen systematischen Platz ein (Heintz und Nadai 1998). Im Gegensatz dazu wird in der ungleichheitstheoretisch geprägten Geschlechterforschung tendenziell die These vertreten, dass die Kategorie Geschlecht, neben anderen, in allen Bereichen der Gesellschaft eine persistente Bedeutung hat. Der Zusammenschluss dieser beiden Denktraditionen in Anwendung an das vorliegende Forschungsthema - ist nicht nur ein spannendes Unternehmen, sondern auch eine lohnende Aufgabe, da beide Gesellschaftsanalysen die empirischen Gegebenheiten einerseits und die bestehenden Ungleichheiten andererseits nicht in allen Einzelheiten stringent erklären können (Heintz und Nadai 1998): Auf der einen Seite hat Geschlecht nicht mehr die gleiche Ordnungsfunktion wie noch vor wenigen Jahrzehnten, auf der anderen Seite sind in vielen Bereichen

Kinderlosigkeit bei Nicht-Akademikerinnen zwischen 13-16 Prozent liegt, bleiben rund die Hälfte der Wissenschaftlerinnen ohne Nachwuchs (Prskawetz et al. 2008). 
geschlechtsspezifische Persistenzen zu beobachten, die mit modernisierungstheoretische Argumente nicht zu erklären sind (Heintz und Nadai 1998).

Das engere Theoriegebäude stützt sich auf die Geschlechterforschung in der Organisationssoziologie (Kanter 1977; Ferguson 1984; Pringle 1989; Acker 1990; Riegraf 1996; Britton 2002; Wilz 2002; Aulenbacher und Riegraf 2010). Organisationen sind in modernen Gesellschaften ein unausweichlicher Bestandteil ihres Daseins (Froschauer 2008; Froschauer 2012). Dabei liegt dieser Arbeit ein Organisationsbegriff zugrunde, der Organisationen als Rahmenbedingung für das Handeln sozialer Akteure versteht (Abraham und Büschges 2009, 64). Die Definition von Organisation lehnt sich dabei an ein breit gefasstes Konzept von Acker (1990; 1994) an und enthält sowohl formale als auch informelle Strukturen. Er bezieht gesellschaftliche Normen und Interpretationen ein und umfasst das Handeln, die Identitäten und die Gefühle der Organisationsmitglieder (siehe Froschauer 2008; Wilz 2013).

Dem Geschlecht in Organisationen wird erstmals in der Arbeit von Kanter (1977) ein bedeutender Stellenwert eingeräumt. Sie analysiert in der Tradition der Weber'schen Bürokratietheorie und mit Bezug auf Befunde der Minderheitenforschung jene Prozesse, die zur Unterrepräsentanz von weiblichen Führungskräften führen. Kanter (1977) geht davon aus, dass die formalen Strukturen moderner Organisationen zweck- und zielorientiert konzipiert sind und sie damit unabhängig von zugeschriebenen Eigenschaften, Merkmalen und Kompetenzen - somit auch unabhängig vom Geschlecht - funktionieren (Aulenbacher und Riegraf 2010). Gleichzeitig macht sie Mechanismen auf informeller Organisationsebene für Geschlechterungleichheiten verantwortlich, welche einen zentralen Grund für die Unterrepräsentanz von Frauen in Machtpositionen darstellen (Riegraf 2013). Die empirisch messbaren Unterschiede erklärt Kanter (1977) über gesellschaftliche Stereotypisierungen und das Nachwirken von vormodernen patriarchalen Strukturen. Aufgrund der Minderheitenposition nehmen Frauen in Führungspositionen die hervorgehobene Stellung von "tokens" ein: «sie sind besonders sichtbar, unterliegen einer besonderen Aufmerksamkeit und werden nicht als Individuen, sondern als Repräsentanten einer sozialen Gruppe wahrgenommen» (Riegraf 2013, 19). Dies bedeutet, dass Leistung immer auch unter dem Aspekt von Geschlechtlichkeit wahrgenommen wird, wobei sich die Situation von Frauen nicht grundsätzlich von anderen Minderheitengruppen unterscheidet (Aulenbacher und Riegraf 2010).

Diese Position bildet den Ausgangspunkt für zahlreiche theoretische Weiterentwicklungen. Ferguson (1984) und Pringle (1989) beispielsweise widersprechen der Position, dass Organisationen grundsätzlich geschlechtsneutral sind und dass der Ansatz von Kanter (1977) die empirischen Gegebenheiten adäquat erfassen kann. In der Reaktion auf ihre Arbeit entwickelt sich die Gegenthese einer vollkommenen Vergeschlechtlichung von Organisation, die alle Organisationsebenen umfasst und Geschlechtszuweisungen nicht mit der Zuweisung zu anderen Minderheitengruppen 
vergleichbar macht (Riegraf 2013, 20). Massgeblich ist hier die Überzeugung, dass Strukturen und Diskurse in Organisationen durch männliche Erfahrungen und Orientierungen bestimmt sind, (in)formelle Organisationsebenen durchziehen und die weibliche Performanz immer in Referenz zur Männlichen bewerten (Pringle 1989, 88; Riegraf 2013).

In neuerer Forschung wird von apodiktischen Grundhaltungen ein Stück weit Abstand genommen. Empirische Studien helfen dabei, ein differenzierteres Bild vom Zusammenhang von Geschlecht und Organisation zu erlangen (Nassehi 1997; Heintz und Nadai 1998; Britton 2002). Diese Studien zeigen, dass sich in denselben Organisationen reduzierte Geschlechterungleichheiten gleichzeitig neben veränderten oder steigenden Geschlechterungleichheiten beobachten lassen (Riegraf 2013, 104). Damit wird eine stärkere Ergebnisoffenheit eingefordert, die es erlaubt «die unterschiedlichen organisationalen Kontexte zu berücksichtigen, in denen Geschlecht zu Geltung kommt oder eben auch nicht und in denen die Herstellung oder gar die die Auflösung von Geschlechter(un)gleichheiten verstärkt oder behindert werden» (Riegraf 2013, 104). Diese Haltung ist forschungsleitend für den vorliegenden Beitrag. Es soll untersucht werden, in welcher Form organisationale Kontexte die Prozesse der Geschlechterdifferenzierung in ungleichheitsrelevante oder irrelevante bzw. positive oder negative Folgen für Frauen überführen. Damit fungiert Geschlecht zum einen als zentrales Element ungleichheitstheoretischer Überlegungen und zum anderen im Sinne modernisierungstheoretischer Argumente.

\section{Datenmaterial und Auswertungsmethode}

Als Erhebungsinstrument dient eine leitfadengestützte, problemzentrierte Befragung von Wissenschaftlerinnen in Österreich (Witzel 2000). Die Frauen wurden retrospektiv zu ihren Berufsbiografien befragt. Die empirische Datengrundlage umfasst insgesamt 22 Interviews. Die Erhebung fand in zwei Zyklen in den Jahren 2012 und 2013 statt. Das Intervall zwischen den Erhebungsphasen wurde zur Prüfung und Verbesserung des Leitfadens genutzt, gemäss der prozesshaften Offenheit qualitativer Forschung (Lamnek 1995).

Die Auswahl der Interviewpartnerinnen basiert auf zuvor definierten theoriegeleiteten Kriterien, die von den teilnehmenden Frauen erfüllt werden mussten. Die zugrunde liegende These der Kontextualität der Geschlechterdifferenz verlangt ein Untersuchungsdesign, in dem berufliche Kontexte systematisch variiert werden, wobei das numerische Geschlechterverhältnis als zentrale Vergleichsdimension dient (Heintz und Nadai 1998, 83). In die Studie wurden dementsprechend Disziplinen integriert, die sich nach ihrer Geschlechtszusammensetzung in Österreich unterscheiden (siehe Tabelle 1). Zusätzlich wurde bei der Auswahl die folgenden Kriterien berücksichtigt: Alle Interviewpartnerinnen sind bzw. waren an einer österreichischen 
Tabelle $1 \quad$ Zusammensetzung des Samples

\begin{tabular}{lll}
\hline Wissenschaftsdisziplin und Geschlechterverhältnis (in \%, M/F) in Österreich & Name & Alter (Jahre) \\
\hline Zum Zeitpunkt des Interviews im Universitätssystem beschäftigt & & \\
Sozialwissenschaften (50/50) & Anna & 52 \\
& Alexandra & 76 \\
Geisteswissenschaften (50/50) & Margit & 40 \\
& Eva & 53 \\
Rechts- \&Wirtschaftswissenschaften (50/50) & Sigrid & 74 \\
& Barbara & 51 \\
Technikwissenschaften (80/20) & Laura & 41 \\
& Katharina & 52 \\
Naturwissenschaften (70/30) & Maria & 62 \\
& Sophia & 61 \\
& Lisa & 46 \\
& Manuela & 57
\end{tabular}

Zum Zeitpunkt des Interviews aus dem Universitätssystem ausgestiegen

Sozialwissenschaften (50/50)

Monika $\quad 33$

Daniela $\quad 38$

Nicole 29

Petra $\quad 30$

Geisteswissenschaften (50/50)

Claudia $\quad 30$

Brigitte $\quad 31$

Martina 29

Rechts-\& Wirtschaftswissenschaften (50/50)

Karin 38

Technikwissenschaften (80/20)

Veronika

Naturwissenschaften (70/30)

Renate

Anmerkungen: Aus Gründen der Anonymität sind alle Namen der Interviewpartnerinnen, und Namen die von den Interviewpartnerinnen in den Gesprächen erwähnt und in den zitierten Passagen genannt werden, geändert. Ausgewiesen werden die prozentualen Anteile des wissenschaftlichen Personals nach Männern und Frauen und Wissenschaftsdisziplinen; nicht enthalten sind Universitäten der Künste.

Quelle: Statistik Austria (2011); eigene Darstellung.

Universität beschäftigt. Die Fallgruppen umfassen zum einen Wissenschaftlerinnen, die aufgrund der Barrieren in verschiedenen beruflichen Positionen aus dem universitären System ausgestiegen sind. Da jene Interviewpartnerinnen nicht von Barrieren berichten können, die sie aufgrund ihres (frühzeitigen) Ausstiegs möglicherweise 
nicht erfahren haben, werden zum anderen Wissenschaftlerinnen in die Untersuchung eingeschlossen, die trotz Barrieren langfristige Berufsbiografien vorweisen.

Der Feldzugang zur ersten Interviewpartnerin erfolgte über einen Kontakt an der Universität Wien. Das Schneeballverfahren wurde eingesetzt, um weitere Wissenschaftlerinnen für die Studie zu gewinnen. Zusätzlich wurden Interviewpartnerinnen gezielt über die Homepages der Institute angeschrieben. Die Interviews wurden entlang von erzählungsgenerierenden Kommunikationsstrategien aufgebaut: Gesprächseinstieg, allgemeine Sondierungen und Ad-hoc-Fragen (Witzel 2000). Nach der vollständigen Transkription der Interviews, wurde das Material zunächst mit der Methode des verlaufsstrukturierten Themenvergleichs (Witzel 2000) ausgewertet. Dabei dienen zuerst zentrale Themen einzelner Fälle und biografische Charakteristika als vorläufige Ergebnisse des theoriegenerierenden Interpretationsschritts; sie werden danach zu komprimierten Aussagen verdichtet, wobei Originaltextstellen und Paraphrasierungen verwendet werden (Witzel 2000). Die speziellen Textbausteine zu den Barrieren für Frauen in der universitären Wissenschaft wurden in einem zweiten Schritt mit dem Verfahren der axialen Kodierung bearbeitet (nach Strauss und Corbin 1996). Beim axialen Kodieren liegt der Fokus darauf, ein Phänomen in Bezug auf die Bedingungen zu spezifizieren die das Phänomen verursachen; auf dem Kontext in dem das Phänomen eingebettet ist; die Handlungs- und interaktionalen Strategien, durch die es bewältigt wird, und die Konsequenzen dieser Strategien (Strauss und Corbin 1996, 76). Dieses kombinierte Auswertungsverfahren erlaubt es, thematische Bündel in den Berufsverläufen der Interviewpartnerinnen zu identifizieren, relevante Barrieren aufzufächern, welche die Unterrepräsentanz von Frauen im Wissenschaftssystem mitbedingen oder Gründe für einen Ausstieg darstellen. Gleichzeitig werden dabei die spezifischen Kontexte in beiden analytischen Schritten berücksichtigt. Der Fallgruppenvergleich untersucht dementsprechend Unterschiede und Gemeinsamkeiten in den Berufsbiografien entlang der ausgewiesenen Barrieren.

\section{$6 \quad$ Ergebnisse der Studie}

Die Analyse des Materials bringt drei zentrale Themenblöcke hervor: (1) Soziale Netzwerke, (2) Praktiken im universitären Arbeitsalltag und (3) die Vereinbarkeit von beruflichen und privaten Verpflichtungen. Zum einen stellen sie entscheidende Barrieren in den Berufsverläufen der Wissenschaftlerinnen dar. Zum anderen sind diese Barrieren gleichzeitig Faktoren, die bei der zweiten Fallgruppe den Ausstieg aus dem universitären Wissenschaftssystem beeinflusst haben. Der Schwerpunkt der Analyse liegt auf den beruflichen Kontexten und den geschlechtsspezifischen Praktiken innerhalb der Organisationen. 


\subsection{Im Netz der Wissenschaft}

Die Analyse des empirischen Materials belegt die hohe Relevanz von sozialen Netzwerken für den Berufsverlauf in der Wissenschaft. Dabei können verschiedene Formen von Netzwerken differenziert werden, die in unterschiedlichen Kontexten prävalent werden. Netzwerke im Sinne eines fachlichen Austausches auf Konferenzen innerhalb einer internationalen wissenschaftlichen Community sind nicht nur förderlich, sondern notwendig für den Berufserfolg. Der Nutzen dieser Netzwerke wird hier auf unterschiedlichen Ebenen verortet: Einerseits ist die Teilnahme an diesen Netzwerken wichtig, um die Qualität der eigenen wissenschaftlichen Arbeit an international etablierten Standards zu orientieren. Andererseits gilt das Netzwerken für zukünftige Kooperationsmöglichkeiten - z. B. in gemeinsamen Forschungsprojekten - als unerlässlich. Hervorgehoben wird von den Interviewpartnerinnen, dass der Schwerpunkt auf dem fachspezifischen Austausch und der Präsentation neuester Forschungsergebnisse liegt; ob Wissenschaftler oder Wissenschaftlerinnen die Befunde vorstellen, spielt eine untergeordnete Rolle. Insgesamt werden diese Kooperationsnetzwerke von beiden Fallgruppen positiv bewertet.

Im Gegensatz dazu thematisieren die Interviewpartnerinnen beider Fallgruppen eine andere Form der Netzwerke. Diese zielen stark auf Exklusivität ab, betonen die Geschlechtshomogenität und operieren auf einer informellen und (über)organisationalen Ebene. Diese Form der Netzwerke wird von den Wissenschaftlerinnen beider Fallgruppen kritisiert. Sie können als Ausschlussnetzwerke beschrieben werden. Hier liefert die Analyse erste Hinweise dafür, dass Geschlecht im Kontext dieser Netzwerkstrukturen weiterhin eine wichtige Ordnungsfunktion besitzt. Anna spricht sich z. B. dafür aus, dass auch bei diesen Netzwerken die Inhalte stärker betont und weniger Gewicht auf die Geschlechtszugehörigkeit gelegt werden sollte. Gleichzeitig verwendet sie die Möglichkeitsform, was darauf hinweist, dass eine gleichberechtigte Situation derzeit noch nicht erreicht ist und ihrerseits auch Skepsis über eine zukünftige Gleichstellung besteht:

\section{Ideal wäre, wenn das Thema "Mann-Frau» kein Thema mehr wäre. Wenn sich wirklich die Geschlechter mischen. Wenn die Sachlichkeit das Thema ist. (Anna, 52 Jahre)}

Die Analyse des Materials verdeutlicht ausserdem, dass diese Form der sozialen Netzwerke den beruflichen Fortschritt beider Fallgruppen erschwert. Daniela ist aus der Wissenschaft ausgestiegen; dabei haben die informellen Netzwerke ihre Entscheidung beeinflusst. Sie erzählt, dass sie Unterschiede in den beruflichen Erfolgen zwischen Männern und Frauen beobachtet hat, was sie auch auf die Rolle von männlich dominierten Netzwerken zurückführt.

Was ich schon gesehen habe, dass einfach die Männer, die sehr gut waren, sind weiter gekommen, als die Frauen, die sehr gut waren. (...) Also ich glaube 
es hat einfach auch viel damit zu tun, mit diesen Männernetzwerken. (...) Die Männer gehen mit den Männern Mittagessen oder die Männer trinken mit den Männern am Abend ein Bier. (Daniela, 38 Jahre)

Diese Interviewpassage verdeutlicht, dass geschlechtlich geprägte Ungleichheiten auf der organisationalen Ebene verhaftet sind und sich über den beruflichen Alltag hinaus fortschreiben. Als Folge werden ungleiche Geschlechterverhältnisse reproduziert, wobei hier die sozial exklusiven Zusammenschlüsse unter Männern auf einer informellen Ebene operieren. Die Analyse zeigt, dass sich diese Netzwerke nachteilig auf die Karrieren von Wissenschaftlerinnen auswirken, da die Teilhabe an diesen informellen Zusammentreffen als eine Informationsquelle für berufliche (Entwicklungs)Möglichkeiten und Karrierechancen gilt. Der Erfolg, den Wissenschaftler durch diese informellen Netzwerke erzielen können, wird von den befragten Frauen darauf zurückgeführt, dass Männer- im Vergleich zu Frauennetzwerken immer noch mehr Einfluss in der wissenschaftlichen Community besitzen:

Netzwerke machen ja nur dann Sinn, wenn sie auch mit Macht und mit Ressourcen ausgestattet sind und viele von diesen Franennetzwerken sind das halt nicht. (Manuela, 57 Jahre)

Ähnliche Ergebnisse erzielen Kjeldal und Kollegen (2005). Sie zeigen, dass männlichdominierte Netzwerke sehr wichtig sind, wenn es um die Verteilung von Ressourcen geht. Interessant ist, dass die Ergebnisse der vorliegenden Studie auf Differenzen zwischen den unterschiedlichen organisationalen oder institutionellen Anbindungen und den disziplinären Hintergründen verweist. (Informelle) Netzwerke spielen in traditionell eher männlich dominierten Wissenschaftsdisziplinen nach wie vor eine grössere Rolle. Vor allem in den Technik- und Naturwissenschaften wird von den Interviewpartnerinnen der vorliegenden Studie vom starken Einfluss der Männernetzwerke berichtet, die teilweise mit bündischen Strukturen oder Seilschaften verglichen werden.

Ein anderer Kontext, in dem soziale Netzwerke eine wichtige Rolle spielen und nachteilige Folgen für Wissenschaftlerinnen hervorrufen, sind Entscheidungssituationen um Führungspositionen. Obwohl Katharina habilitiert ist und damit einen erfolgreichen Karriereweg in einem männlich dominierten Umfeld vorweist, hat sie den Einfluss der Netzwerke bisher unterschätzt. Erst durch die Bewerbung für eine leitende Position ist sie mit dieser Form der Männernetzwerke konfrontiert worden.

Von denen ich bis vor drei Wochen nie geglaubt hätte, wie stark die sind. Die Männernetzwerke. Aber seit dieser heissen Phase zur Wahl weiss ich: Die sind wirklich existent. Also ich habe das vorher nie gespürt. (Katharina, 52 Jahre)

Die Einflussnahme der Netzwerke entsteht durch eine Entscheidungssituation - die Wahl zu einer hierarchisch höher gestellten Position in einer traditionell männlich dominierten Organisation. Ein Urteil über Netzwerke, das Katharina auf langjährige 
Erfahrung stützt, muss aufgrund eines intensiven Ereignisses revidiert werden («nie» hätte Katharina das geglaubt; "nie» hatte Katharina das zuvor gespürt). Dies gibt Aufschluss darüber, wie stark der Einfluss in dieser Entscheidungssituation gewesen ist. Ähnlich wie zuvor Daniela hebt sie die Relevanz von informellen Gesprächen hervor. Solche Gespräche über Katharina als Frau und nicht über Katharina als (un)geeignete Wissenschaftlerin für die ausgeschriebene Position rücken in dieser Situation ins Zentrum.

Und in den letzten Wochen habe ich gelernt, dass ein offenes Gespräch gar nicht mehr ging, sondern dass nur mehr auf dieser informellen Ebene geredet wurde und dann - ich sag's mal so - recht unsachlich geredet wurde. (...) So eine emotionale Ebene, wo Gerüchte geschürt worden sind, wo dann völlig unklar war, gibt es ein Problem und wenn ja, wo ist das überhaupt, und was hat das überhaupt mit mir zu tun. (...) Weil manche Diskussionen schon untergriffg sind und auf die Rolle als Frau anspielen. (...) So eine Diskussion gibt's über Männern nicht. (Katharina, 52 Jahre)

Hier entsteht ein spezieller Mechanismus der den sozialen Ausschluss bezweckt und der einen mikropolitischen Wechsel der Umgangsformen anstrebt. Es geht in diesen informellen Gesprächen nicht mehr um Katharinas Leistungen oder Fähigkeiten für den Job, sondern vielmehr um ihre Rolle als Frau in dieser Position. ${ }^{7}$ Gleichzeitig werden diese Gespräche auf eine emotionale Ebene gehoben, die eine Entgegnung erschweren. Obwohl Katharina betont, dass sowohl Frauen und Männer in Führungspositionen alleine aufgrund der Position mit diesen Kommunikationsformen konfrontiert sind, würde zusätzlich noch ein geschlechtsspezifischer Unterschied bestehen. Sie geht davon aus, dass es ähnlich informell «untergriffige» Gespräche über Männer in derselben Position nicht gibt. Interessant ist dabei nicht nur, dass die Wahl von Katharina in diese Führungsposition in einer traditionell männlichdominierten Disziplin und einer bisher männlich geführten Organisation stattfindet. An diesem Beispiel deutet sich auch an, dass sich innerhalb ein und derselben Organisation neben persistenten Geschlechterungleichheiten nach und nach auch ungleichheitsrelativierende Prozesse durchsetzen, die im Sinne einer modernisierungstheoretischen Perspektive interpretiert werden könnten (vgl. Heintz und Nadai 1998; Riegraf 2013).

Darüber hinaus verweist die Analyse meiner Studie auf organisationale Kontexte, in denen soziale Netzwerke positive Folgen für Wissenschaftlerinnen haben. Der exklusive Zusammenschluss unter Frauen wird als Umfeld etabliert, in dem gegenseitige Unterstützung und Anteilnahme vorherrschen. Dabei entsteht ein Raum, der den Aufbau anerkennender und wertschätzender Beziehungen fördert (Bock 2010). Diese Form der Vernetzung unter den Wissenschaftlerinnen besitzt

$7 \quad$ In Anlehnung an Ferguson (1984) und Pringle (1989) weisen diese Routinen bei Rekrutierungsprozessen auf vergeschlechtlichte Organisationen hin (vgl. Kapitel 6.2). 
eine Funktion der Bemächtigung - des Empowerments -, etabliert Solidarität unter den Frauen und wirkt damit einer individualisierten Bewältigung von strukturellen Problemen und Barrieren entgegen. Zudem übernehmen erfahrene Frauen Mentoring-Aufgaben für Nachwuchswissenschaftlerinnen. Manuela verfügt als Professorin über eine langjährige Berufserfahrung; sie bewertet diese Form des Netzwerkens als essenziell für weibliche Wissenschaftskarrieren:

Dass man sich einmal hinsetzt und sagt, ja was wollen wir eigentlich, wohin wollen wir, wie erreichen wir das. (Manuela, 57 Jahre)

Verbunden mit Erwartungen über die Stärkung des politischen Einflusses, werden in diesem Kontext Frauennetzwerke als Bewältigungsstrategie aufgebaut (Chen 2001). Um eine Verbesserung der derzeitigen Situation an den Universitäten zu erreichen, werden geschlechterpolitische Zielsetzungen formuliert und durchgesetzt, die individuell schwer zu erreichen sind (Bock 2010).

Dabei wird der ausschliessende Mechanismus der Geschlechtshomogenität als (Gegen)Strategie zur Förderung von (Nachwuchs)Wissenschaftlerinnen übernommen. Monika berichtet von einer konkreten Initiative zur Vernetzung innerhalb ihrer Fakultät, welche auf einer überorganisationalen Ebene institutionalisiert wurde, um den (wissenschaftlichen) Austausch und Kooperationsmöglichkeiten zu fördern.

Ganz konkret hat eine Kollegin ein Vernetzungsfrühstück (...) ins Leben gerufen, wo eben nur Frauen die in der Wissenschaft tätig sind (...), um da zu schauen, was machen die einzelnen Frauen und wie kann man da auch kooperieren. Also ganz konkret auch unter Ausschluss der Männer. Um da gewisse Ressourcen auch auszuschöpfen und eben Networking unter den Frauen zu betreiben. (Monika, 33 Jahre)

Dabei geht es neben der Bemächtigungsfunktion und einer Solidarisierung innerhalb der Gruppe der Wissenschaftlerinnen konkret um den fachlichen Austausch und die Erschliessung von Ressourcen, um Wissenschaftlerinnen bei Positionsverbesserungen oder Statusgewinnen zu unterstützen (Bock 2010). Parallelen sind hier zur "bounded-solidarity" zu sehen, die aufgrund einer gemeinsam erlebten Situation Gruppennormen und gegenseitige Unterstützung etabliert (Portes und Sensenbrenner 1993).

\subsection{Praktiken im universitären Arbeitsalltag}

Der universitäre Arbeitsalltag ist von spezifischen Praktiken geprägt, bei denen verschiedene Aspekte von vergeschlechtlichen Organisationen zum Teil sehr deutlich werden. Von hoher Relevanz sind dabei die Routinen des Rekrutierens und des Förderns - insbesondere im Bereich der Nachwuchsförderung. In Sigrids Berufsbiografie zeigt sich deutlich, dass sie durch geschlechtsspezifische Praktiken des Förderns Nachteile erfahren hat, denen sie aktiv entgegenwirken musste. Eine erfolgreiche 
Wissenschaftskarriere hat sie nur aus eigenem Antrieb und trotz massiver Barrieren erreicht. Zuspruch und Anerkennung vonseiten ihres Vorgesetzten hat Sigrid erst erfahren, nachdem sie sich als erfolgreiche Wissenschaftlerin etablieren konnte.

Die Schwierigkeiten, die ich mit ihm [dem Vorgesetzten] hatte, waren nicht einmal so sehr wissenschaftlicher Natur, weil er hat ja durchaus meine Arbeit geschätzt, aber persönlicher Natur. Also eine Frau im wissenschaftlichen Bereich, noch dazu auf Augenhöhe, das geht nicht. Es war dann allerdings so, wie ich mich durchgeboxt hatte, und mit meinem ersten Buch und dann mit meinem zweiten Buch Erfolg hatte, dann war er auf einmal stolz auf mich und hatte mich ja immer gefördert gehabt. (Sigrid, 74 Jahre)

Monika erzählt ebenfalls von geschlechtsspezifischen Praktiken der Nachwuchsförderung. Sie hat gemeinsam mit anderen NachwuchswissenschaftlerInnen in einem Drei-Jahres-Drittmittelprojekt gearbeitet. Bereits vor Auslaufen des Projektes wurde die intensive Zusammenarbeit reduziert.

Die haben uns jetzt dann nicht mehr gebraucht. Wir haben denen ja schon die ganzen Dateninfos geliefert (...). Und wir waren dann schon wie ausgepresst und weggeschmissen. Das war ja auch klar: Unser Projekt läuft ja dann aus; wozu sollen wir noch investieren in die jungen Damen. Und schon vor Ablauf der Zeit ist man irgendwie auf das Abstellgleis gesetzt worden. Während beim Peter [einem Kollegen] (...) da haben sie anscheinend mehr Potential erkannt, mit dem dann noch bei anderen Projekten vielleicht weiter zu machen. (Monika, 33 Jahre)

Monika fühlt sich als Person und Arbeitskraft ausgenutzt - «ausgepresst und weggeschmissen». Sie kritisiert, dass ihrem männlichen Kollegen eine bessere Position innerhalb der Organisation zugeschrieben wurde, obwohl beide formal gleich gestellt sind. Eine Zusammenarbeit in weiterführenden Projekten wird nur ihrem Kollegen angeboten.

Ein weiteres Charakteristikum des Arbeitsalltags ist das hohe Mass an Instabilität. Um in der universitären Wissenschaft eine erfolgreiche Karriere aufzubauen, muss ein langfristiges Arrangement in Unsicherheit, Flexibilitätsbereitschaft und kurzfristigen Perspektiven akzeptiert werden (Dörre und Neis 2008; Fritsch 2014). Die vorliegende Analyse belegt, dass die Unsicherheit in allen Berufsbiografien einen wichtigen Stellenwert einnimmt. Dabei erstrecken sich diese Phasen der Unsicherheit - anders als in anderen Bereichen des Arbeitsmarktes - über weite Strecken der beruflichen Laufbahn.

Gerade wenn man an der Uni anfängt zu arbeiten, bis man wirklich davon leben kann, das ist ja ein langer Weg. (Claudia, 30 Jahre) 
Kurzfristige Beschäftigungsverhältnisse sind auch hier insbesondere bei den Nachwuchswissenschaftlerinnen ein zentrales Thema. Die Unsicherheit und die begrenzte Anzahl der Stellen vor allem auf den weiterführenden Positionen erschweren nicht nur den persönlichen beruflichen Fortschritt, sondern bieten zunehmend mehr Raum für eine allgemeine Perspektivenlosigkeit.

\section{[Es] wird geredet von Förderung der Nachwuchswissenschaftlerin bis hin zu «die Exzellenz». (...) Es gibt viele Möglichkeiten und auch Finanzierungs- quellen, wo Dissertantinnen und Prae-Docs gefördert werden. Aber was ist danach? Und das ist ja auch den Instituten und den Chefs dort egal. (...) Da passiert einfach nachher nichts. Das ärgert mich einfach. (Monika, 33 Jahre)}

Die Nachwuchsförderung ist wenig strukturiert und vor allem vom persönlichen Engagement der/des Vorgesetzten abhängig. Der Mangel an Stellen und der unsystematische Prozess des Förderns wird im wissenschaftlichen Diskurs unter dem Begriff des "bottleneck" verhandelt (Müller 2012). Er beschreibt zwei wesentliche Übergänge (Phase bis zur und nach der Promotion) in akademischen Berufsverläufen. Da die Anzahl der HochschulabsolventInnen steigt, die Zahl der zur Verfügung stehenden Ausbildungspositionen aber gleichzeitig nicht im selben Ausmass wächst oder in manchen Bereichen sogar zurück geht, erhöht sich der Wettbewerbsdruck um diese Stellen zunehmend. Dabei liegt es nahe, dass Wissenschaftlerinnen an diesen Schnittstellen durch die geschlechtsspezifischen Praktiken des Förderns im allgemeinen Wettbewerb tendenziell mit Nachteilen konfrontiert sind.

Die instabilen Beschäftigungsverhältnisse werden von einer Mobilitäts- und Flexibilitätsbereitschaft begleitet, welche die gesamte Biografie prägt. Einige der befragten Wissenschaftlerinnen geben an, dass sie nicht mehr bereit sind, die Wissenschaft über alle anderen Lebensbereiche zu stellen, sich in regelmässigen Abständen neu zu orientieren und nach wenigen Jahren die Lebensmittelpunkte neu zu gestalten. Brigitte ist zum Zeitpunkt des Interviews ohne Beschäftigung und beendet ihre Dissertation ohne Finanzierung. Sie thematisiert die hohe berufliche Unsicherheit als wahrscheinlich wichtigste Barriere für sie und andere NachwuchswissenschaftlerInnen.

Ja sicher, das [die Unsicherheit] ist ein grosses Thema. (...) Weil ich sehe es schon so: Wenn ich wirklich in der Wissenschaft weiter mache, dann werde ich nicht ortsgebunden sein können. Also da muss ich flexibel sein. Mal für ein paar Jahre dorthin gehen vielleicht. Also es ist eher unwahrscheinlich, dass ich dann in [einer Stadt] bleiben könnte. (Brigitte, 31 Jahre)

Einsatzbereitschaft, Arbeitsüberlastung und ein hoher Leistungsdruck spielen im universitären Arbeitsalltag ebenfalls eine zentrale Rolle. Zum einen wird eine ausgeprägte zeitliche Verfügbarkeit verlangt. 
Und wie wird Wissenschaft jetzt gelebt? Das ist eher diese Rund-um-dieUhr-Verfügbarkeit. (Laura, 41 Jahre)

Dabei liefern die Ergebnisse der vorliegenden Studie erste Hinweise dafür, dass in diesem Zusammenhang langsam eine neue, ganz spezielle Form der Vernetzung innerhalb der NachwuchswissenschaftlerInnen beginnt, die eine kollektive Auseinandersetzung auf einer überorganisationalen Ebene fördert und gemeinschaftlichen Austausch - auch mit Hilfe neuer Medientechnologien - aufbaut. Obwohl die folgende Interviewpassage die (immer noch) überwiegende Vorstellung von der Wissenschaft als «alles-unterordnende Lebensform» verdeutlicht, zeigt sich zumindest auch, dass hier strukturelle Probleme ein kollektives Problembewusstsein formieren und nicht mehr nur ausschliesslich individualisiert bewältigt werden müssen.

Ich lese es auch öfter immer wieder auf Twitter. Da gibt es unter PhDChat (...). Und da habe ich vor kurzem auch gelesen: (...) Ob man über die Stunden hinaus arbeiten sollte. Da haben Leute dann schon irgendwie geschrieben: "Das gehört irgendwie dazu zum Wissenschaftler sein. Dass es nicht mit dem Ende der acht Stunden am Tag aus ist, weil sonst ist man kein richtiger Wissenschaftler." Also ich bin was das angeht ein bisschen ambivalent, weil ich kenne so Momente, wo das total schön sein kann. Aber ich kenne es auch, dass es eine Belastung ist. (Brigitte, 31 Jahre)

Zum anderen herrscht im Arbeitsalltag der interviewten Wissenschaftlerinnen ein Erwartungsdruck vor, in nahezu allen Bereichen nicht nur sehr gut, sondern hervorragend zu sein. Wissenschaftliche Arbeit an Universitäten findet unter kompetitiven Rahmenbedingungen statt. Der Anspruch, international wettbewerbsfähig zu sein und laufend Drittmittelprojekte zu akquirieren, um fehlende Grundfinanzierungen oder Beschäftigungsverhältnisse auszugleichen, nimmt in den letzten Jahren deutlich zu (vgl. Klecha und Reimer 2008). Unabhängig von der realen Notwendigkeit, hohe Qualitätsstandards einzuhalten, wird die tägliche Arbeit von einem konstanten Erwartungsdruck begleitet. Daniela beschreibt, dass dieser Druck nicht nur das Gegenteil bewirken und im Einzelfall zum Stillstand führen kann, sondern auch Auswirkungen auf ihre physische Gesundheit hatte:

Der ständige Druck, der da gemacht worden ist, hat mich mehr gelähmt, als dass er mich gepusht hätte. Und das hat mir nicht gut getan, auch gesundheitlich. Und dann muss man was verändern. (...) Dieser Druck: Man muss ständig publizieren, man muss ständig der Beste sein. Es genügt nicht in einem Peer-Reviewed Journal, sondern es muss ein TOP-Peer-Reviewed Journal sein. Das war einfach zu viel. Oder da wollt' ich dann einfach nicht mehr mit. (Daniela, 38 Jahre)

Daniela spricht hier von einem ständigen Leistungsdruck, der unabhängig von den tatsächlichen Erfolgen oder Misserfolgen in der Wissenschaft präsent ist. Da das 
Ziel, «der Beste zu sein», in diesem System unerreichbar scheint, kann die Resignation bzw. ein Ausstieg aus dem Universitätssystem ein möglicher Ausweg sein. Interessant ist dabei, dass die Daniela die Formulierung «man muss ständig der Beste sein» verwendet. Es scheint, dass hier das Selbstverständnis der Frauen in Bezug zu einer männlichen Norm definiert wird bzw. definiert werden muss, was somit auf die Prozesse einer vergeschlechtlichen Organisation aufmerksam macht.

\section{3 "Work-Life \& Work Family Balance" - ein Widerspruch in der Wissenschaft?}

Die Analyse des Materials zeigt, dass «Beruf und Familie» häufiger als binär kodiertes Gegensatzpaar aufgefasst wird und die Wissenschaftlerinnen damit in ihrer beruflichen Laufbahn vor eine Entweder-Oder-Entscheidung stellt. Als mögliche Handlungsoptionen gelten hier ein Ausstieg aus der Wissenschaft oder keine Kinder zu bekommen. Lisa hat eine erfolgreiche Wissenschaftskarriere schon früh als persönliches Ziel definiert und sich deswegen bewusst gegen Kinder entschieden.

\section{Aber ich täte mich nicht so leicht, wenn ich eine Familie hätte. Also das muss ich klar sagen, also wenn Kinder da sind, ist das schwieriger. (...) Ja, viele Frauen haben kein Kind. So wie ich. Dann ist es leichter. (Lisa, 46 Jahre)}

Andere Interviewpartnerinnen haben in ihrer Berufslaufbahn Kinder bekommen. Sie berichten von unterschiedlichen Schwierigkeiten, die durch die Doppelbelastung entstehen. Für Katharina ist die Familiengründung die «einzige Krise überhaupt» in ihrer Karriere als Wissenschaftlerin.

Und habe dann nach einem Monat oder so nach Arbeitsbeginn gemerkt, dass ich schwanger bin. (...) Und als ich dann das zweite Mal schwanger war, war dann schon die Frage: Kriegt man das noch unter einen Hut. Das war so die einzige Krise, die ich für mich überhaupt erlebt habe. (...) Und das ist aber glaube ich etwas, das für jede Fran auf irgendeine Art und Weise sehr bedeutsam ist. (Katharina, 52 Jahre)

In der Literatur wird die Doppelbelastung durch Beruf und Familie als zentrale Ursache für die Unterrepräsentanz von Frauen auf den höheren Positionsstufen diskutiert (Raddon 2002; Abele 2003; Acker und Armenti 2004). Katharina macht dabei jedoch deutlich, dass vor allem die Geburt des zweiten Kindes existenzielle Fragen aufwirft. Die Formulierung «unter einen Hut kriegen» verweist darauf, dass Katharina nicht daran zweifelt, den Herausforderungen jeweils für sich gewachsen zu sein. Vielmehr geht es darum, dass beide Bereiche (aufgrund der Zeitintensivität) schwierig zu vereinbaren sind.

In diesem Zusammenhang beschreibt Sophia die Situation zur Vereinbarkeit von beruflichen Verpflichtungen als Wissenschaftlerin und privaten Verpflichtungen als Mutter sogar noch drastischer. 
Das heisst die schwierigste Zeit, das ist glaub ich ganz typisch für Frauenkarrieren (...), sind die Höllenjahre zwischen 30 und 40. Da muss man einfach durch. (...) Und in den 30er Jahren ist es für Frauen besonders schwer, weil da ist die Frage, Familie, Karriere, wie etablier ich mich? (...) Und ich find ab 40 (...), da entspannt sich dann das Leben. (Sophia, 61 Jahre)

«Die Höllenjahre zwischen 30 und 40» sind in Sophias Einschätzung etwas, was vor allem in den Biografien von Frauen eine Rolle spielt, da es zu gleichen Teilen darum geht, eine wissenschaftliche Karriere und eine Familie aufzubauen, sowie gleichzeitig die Unsicherheiten der universitären Berufsverläufe zu meistern. Obwohl für sie diese Zeit sehr belastend ist, steigt Sophia nicht aus der Wissenschaft aus. Mangels eines allgemeinen Problembewusstseins für diese speziell weibliche Barriere und einer fehlenden Vernetzung innerhalb der universitären Community bewältigt sie diese Phase in erster Linie durch eine extensive Form der Selbstausbeutung. Zusätzlich macht sie deutlich, wie stark verknüpft die Belastungen sind. Ist diese Phase überstanden, entspannt sich das gesamte Leben.

In diesem Zusammenhang beschreibt Laura eine Entwicklung, die sie erst in den letzten Jahren beobachtet und selbst in ihrem eigenen Arbeitsbereich versucht zu etablieren. Es formiert sich langsam ein kollektives Problembewusstsein für das Thema der Vereinbarkeit bzw. "Work-Life-Balance", das die traditionellen Praktiken der Arbeitsorganisation und des Arbeitsethos kritisiert und gleichzeitig nicht ausschliesslich mit betreuungspflichtigen Kindern oder familiären Verpflichtungen assoziiert.

Und wir versuchen langsam auch eine Kultur zu etablieren und zu sensibilisieren, dass z. B. Sitzungstermine nach einer bestimmten Tageszeit nicht mehr unbedingt gelegt werden sollten (...). Es hat aber sehr lange das Bild gegeben, (...) ein Wissenschaftler-und jetzt bewusst männlich formuliert-, das ist ein Mensch, der lebt ja nur für die Wissenschaft. (...) Das Spannende an dem Punkt ist, (...) dass es nicht der Lebensrealität und den Wünschen der Personen entspricht, wenn man das ändern würde, ist es nicht nur vordergründig aus dem Anliegen der Frauenförderung heraus, sondern es wäre eine Kultur, die beiden Geschlechtern zugutekommt. (Laura, 41 Jahre)

Hier wird eine Wissenschaft kritisiert, die als Lebensform zu verstehen ist, die den privaten Lebensraum der wissenschaftlichen Forschung unterordnet und das gesamte Leben prägt (vgl. Weber 1919). Interessant ist dabei, dass ein Aufbrechen des traditionellen Verständnisses von Wissenschaft - und damit das Aufbrechen der etablierten Formen der Arbeitsorganisation - nicht ausschliesslich aus den Interessen der Frauenbewegung entspringt. Eine Reorganisation könnte Vorteile sowohl für Wissenschaftlerinnen als auch für Wissenschaftler nach sich ziehen.

Darüber hinaus wird die Vereinbarkeit von beruflichen und privaten Verpflichtungen auch hier durch die prekären Arbeitsbedingungen in der universitären Wis- 
senschaft erschwert (Kahlert 2013, 141-143). Es zeigt sich, dass die hohe berufliche Unsicherheit von den Interviewpartnerinnen beider Fallgruppen als zentrale Barriere gerade in der Phase der Familiengründung thematisiert wird. Gleichzeitig können Erwartungen über zukünftige Vereinbarkeitsprobleme die wichtigen Schlüsselstellen im Berufsverlauf beeinflussen. Die Beschäftigungsbedingungen lösen bei einigen Interviewpartnerinnen der zweiten Fallgruppe eine Form der Perspektivenlosigkeit aus, die verbunden mit antizipierten Vereinbarkeitsproblemen eine Entscheidung für einen Ausstieg aus der Wissenschaft bedingen:

Ich habe mich nicht wohl gefühlt mit dieser Perspektivenlosigkeit. (...) Und bei mir war's dann konkret auch das Denken: Ich möchte dann irgendwann einmal Familie gründen und da kann ich diese prekären Beschäftigungsverhältnisse nicht brauchen. (...) Ich möchte einfach einen unbefristeten Job einmal haben, von dem aus ich erstens schwanger werden kann und zweitens vielleicht auch die Möglichkeit habe, wieder zurück zu kommen. (...) Das war das Ziel für mich, diese Sicherheit und Stabilität zu kriegen, um mein Leben planen zu können. (Monika, 33 Jahre)

Monika ist als wissenschaftliche Assistentin aus dem Universitätssystem ausgestiegen. Stabilität und Sicherheit sind für sie wichtige Aspekte, um die Verbindung zwischen den beiden Bereichen "Beruf und Privat» planen zu können. Die universitäre Wissenschaft bietet diese Voraussetzungen aus ihrer Sicht nicht.

Und da bin ich auch nicht bereit [hier Abstriche zu machen]. Also erstens möchte ich Kinder haben und zweitens möchte ich dann aber auch Zeit für die Kinder haben. Und da habe ich das Gefühl, da geht es sehr selbstausbeuterisch zu. Und da habe ich schon auch manchmal das Gefühl, als müssen Frauen dann noch einmal eine Spur mehr leisten und zeigen was sie können, um sich in dem Feld da behaupten zu können. (Monika, 33 Jahre)

Monika ist nicht bereit, die «Höllenjahre» von denen Sophie zuvor gesprochen hat, auf sich zu nehmen. Dabei trifft sie die Entscheidung, aus der universitären Wissenschaft auszusteigen, ohne die Vereinbarkeitsproblematik persönlich erlebt zu haben. ${ }^{8}$

Letztlich betonen die Interviewpartnerinnen der zweiten Fallgruppe, dass der zunehmende Druck zur geografischen Mobilität die Vereinbarkeit von Beruf und Familie erschwert. Daniela [38 Jahre] führt den «Zwang ständig mobil zu sein» als zentrale Barriere für weibliche Karrieren und wichtigsten Grund für ihren Ausstieg aus der Wissenschaft an. In ihrer Einschätzung ist die Forderung nach Flexibilität und Mobilität in der Wissenschaft nur sehr schwer mit der notwendigen Stabilität im Privatleben zu vereinbaren (Fritsch 2014). Auch Monika führt die Mobilitätsan-

8 In den Arbeiten von Merton (1948) zu wissenschaftlichen Normen prägt er den Begriff der "self-fulfilling prophecy" als Erklärungsmechanismus für Auswirkungen von Einstellungen oder Handlungen. Prognosen, Vorurteile oder erwartetes Verhalten können sich gemäss dem ThomasTheorem "If men define situations as real, they are real in their consequences" selbst erfüllen. 
forderungen als zentrale Barriere für Frauen an. Monika ist zukünftig nicht mehr bereit, die «Zwangsmassnahme Ausland» zu absolvieren, da eine Familiengründung für sie Vorrang hat und mit Auslandsaufenthalten nicht zu vereinbaren ist.

Im Zuge der Dissertation war es auch Bedingung eigentlich, dass man ins Ausland geht. (...) Und das war dann halt eher so ein Muss. (...) Und eigentlich hat es mir in mein Leben nicht so hineingepasst. Aber gut, dann habe ich halt auch noch 4-5 Monate [im Ausland] verbracht. Jetzt ist aber auch gut für mich. Das interessiert mich jetzt für die nächste Zeit weniger. Aber auch weil für mich jetzt demnächst Familienplanung ansteht. (Monika, 33 Jahre)

Neben den Schwierigkeiten sprechen die Interviewpartnerinnen der ersten Fallgruppe auch von positiven Aspekten. So zum Beispiel Anna. Sie ist froh, das «Kinderkriegen in ihrem biologischen Programm zu haben» und betont, dass es bei dieser Diskussion noch stärker um die strukturelle Unterstützung bei der Karriereentwicklung von Müttern in der Phase der Kleinkindbetreuung und um eine Sensibilisierung für geschlechtsspezifische Prozesse in den unterschiedlichen Organisationen gehen müsse.

Ich finde, wir Frauen haben - Gott sei Dank sag' ich jetzt als Frau und Mutter-haben wir das Kinderkriegen in unserem biologischen Programm. Das würde ich auch nicht hergeben wollen. (...) Daher denke ich, muss die Diskussion noch viel weiter gehen: Wie kann man Frauen, die in der Babypause sind noch mehr in ihrer Karriereentwicklung unterstützen? (Anna, 52 Jahre)

Die Analyse verweist auf weitere positive Auswirkungen auf die Arbeit als Wissenschaftlerin. In erster Linie werden eine Effizienzsteigerung, ein besseres Zeitmanagement, eine stärkere Zielorientierung und Fokussierung betont. Margit organisiert ihre Zeitressourcen jetzt effizienter:

Dass man einfach unglaublich effizient wird (...). Und sich dann weniger verzettelt. Weil man hat einfach nicht die Zeit. Man will sicher nicht vom Kind absparen. Was vielleicht früher anders war, wenn man dann halt nicht zum Sport kommt oder doch nicht ins Kino kommt. (Margit, 40 Jahre)

Margit thematisiert dabei auch, dass sie erst durch ihr Kind eine Form der Gelassenheit und Zuversichtlichkeit gewonnen hat, die ihr in ihrer Arbeit als Wissenschaftlerin nützlich ist.

Aufjeden Fall ist ein Kind zu haben nochmal ein grosser Teil, der zu mehr Gelassenheit führt gegenüber anderen Themen. (...) Ein Kind ist noch viel ein grösserer Impuls, dass einfach Schwierigkeiten im Leben anders gemeistert werden und gar nicht so eine Dramatik annehmen und dadurch meistert man sie auch besser. (Margit, 40 Jahre) 
Viele Interviewpartnerinnen sprechen davon, dass Kinder einen Perspektivenwechsel auslösen, der dabei hilft Prioritäten zu definieren bzw. Stress abzubauen. Durch eine gelassene Herangehensweise und eine zuversichtliche Haltung, können Arbeitsprozesse effizienter und unkomplizierter gestaltet werden. Zusätzlich gewinnen die Wissenschaftlerinnen eine Distanz zum Arbeitsalltag, was eine Aussenperspektive schärft und für das wissenschaftliche Arbeiten nützlich ist.

\section{Diskussion}

Trotz hervorragender Bildungsbiografien und der Implementierung von flächendeckenden Förderungsmassnahmen sind in Österreich immer noch deutlich weniger Wissenschaftlerinnen in höheren Positionsstufen zu finden als Wissenschaftler. Woran liegt es, dass Wissenschaftlerinnen ihre männlichen Kollegen im Qualifikationsprofil teilweise überholt haben, aber dennoch meist weniger erfolgreiche Berufskarrieren vorweisen können? Dieser Widerspruch stellt den Ausgangspunkt des vorliegenden Beitrags dar. Im Zentrum des Untersuchungsinteresses steht die Analyse von Barrieren für Frauen im universitären Wissenschaftssystem in unterschiedlichen organisationalen Kontexten.

Dabei werden die Ergebnisse der empirischen Studie mit Bezug auf modernisierungs- und ungleichheitstheoretischen Prämissen diskutiert. Aktuelle Trends in Österreich belegen, dass geschlechtsspezifische Persistenzen und Reproduktionsmuster ungleicher Geschlechterverhältnisse gleichzeitig neben Modernisierungsprozessen bestehen: Während einerseits vergleichsweise noch nie so viele Frauen in der universitären Wissenschaft tätig waren wie heute, sind andererseits die höheren beruflichen Positionen und spezielle disziplinäre Bereiche nach wie vor fast ausschliesslich männliche Domänen (uni:data 2014). Dieser Beitrag schliesst damit an die Arbeit von Heintz und Nadai (1998) an. In ihrem Kern wird argumentiert, dass es im Zuge der stärkeren Inklusion von Frauen in alle gesellschaftlichen Teilbereiche zu einer Verschiebung der Reproduktionsmechanismen kommt. Anstelle von formalen Ausschlusskriterien wird geschlechtliche Differenzierung in der universitären Wissenschaft heute zunehmend über indirekte und informelle Mechanismen hergestellt, womit die unterschiedlichen organisationalen Kontexte an Relevanz für eine systematische Untersuchung gewinnen (Heintz und Nadai 1998, 88). Die vorliegende Studie versucht, dieses Desiderat einzulösen und präsentiert durch die Analysearbeit neue Perspektiven der geschlechtlichen Differenzierung. Deutlich wird dies insbesondere bei den geschlechtsspezifischen Praktiken des Förderns und des Rekrutierens.

Die Studie zeigt ausserdem, dass (informelle) Netzwerke und die Verbindung zwischen beruflichen und privaten Verpflichtungen bei der gleichzeitigen Notwendigkeit zur Mobilität kritische Phasen in den Lebensentwürfen der Wissenschaft- 
lerinnen darstellen. Der Beitrag verdeutlicht jedoch auch, dass die verschiedenen organisationalen Kontexte nicht ausschliesslich negative Folgen für Wissenschaftlerinnen produzieren. Zukünftige Forschungen könnten hier anknüpfen und sich noch stärker mit den Ambivalenzen, aber auch den positiven Aspekten einer Wissenschaftskarriere auseinander setzen.

\section{Literaturverzeichnis}

Abele, Andrea. 2003. Beruf - kein Problem, Karriere - schon schwieriger: Berufslaufbahnen von Akademikerinnen und Akademikern im Vergleich. S. 147-172 in Frauen und Männer in akademischen Professionen, hrsg. von Andrea Abele, Ernst-Hartmut Hoff und Hans-Uwe Hohner. Heidelberg: Asanger-Verlag.

Abraham, Martin und Günter Büschges. 2009. Einführung in die Organisationssoziologie. Wiesbaden: VS.

Acker, Joan. 1990. Hierarchies, jobs, bodies: A theory of gendered organizations. Gender \& Society 4(2): 139-158.

Acker, Joan. 1994. The gender regime of Swedish banks. Scandinavian Journal of Management 10(2): 117-130.

Acker, Sandra und Carmen Armenti. 2004. Sleepless in academia. Gender and Education 16(1): 3-24.

Aulenbacher, Brigitte. 2010. Gesellschaftsanalysen der Geschlechterforschung. S. 33-58 in Soziologische Geschlechterforschung, hrsg. von Brigitte Aulenbacher, Michael Meuser und Birgit Riegraf. Wiesbanden: VS.

Aulenbacher, Brigitte und Birgit Riegraf. 2010.Geschlechterdifferenzen und Ungleichheiten in Organisationen. S. 157-171 in Soziologische Geschlechterforschung, hrsg. von Brigitte Aulenbacher, Michael Meuser und Birgit Riegraf. Wiesbaden: VS.

Becker, Ruth, Ellen Hilf, Shih-Cheng Lien, Kerstin Köhler, Bärbel Meschkutat, Darja Reuschke und Cornelia Tippel. 2011. Bleiben oder gehen? S. 21-63 in Berufliche Karrieren von Frauen, hrsg. von Waltraud Cornelißen, Alessandra Rusconi und Ruth Becker. Wiesbaden: VS.

Benschop, Yvonne und Margo Bourns. 2003. Crumbling ivory towers: Academic organizing and its gender effects. Gender, Work and Organization 10(2): 194-212.

Bielby, William. 2000. Geschlecht und Karriere: Ist die Wissenschaft ein Sonderfall? S. 55-82 in Wissenschaftskultur und Geschlechterordnung, hrsg. von Beate Krais. Frankfurt a. M.: Campus.

Blickenstaff, Jacob. 2005. Women and science careers: Leaky pipeline or gender filter? Gender and Education 17(4): 369-386.

Bock, Stephanie. 2010. Frauennetzwerke: Geschlechterpolitische Strategie oder exklusive Expertinnennetze? S. 870-878 in Handbuch Frauen- und Geschlechterforschung, hrsg. von Ruth Becker und Beate Kortendiek. Wiesbaden: VS.

Brink, van den Marieke und Yvonne Benschop. 2012. Gender practices in the construction of academic excellence: Sheep with five legs. Organization 19(4): 507-524.

Britton, Dana. 2002. The epistemology of the gendered organization. Gender \& Society 14(3): 418-438.

Buber, Isabella, Caroline Berghammer und Alexia Prskawetz. 2011. Doing Science, ForgoingChildbearing? Evidence from a Sample of Female Scientists in Austria. Vienna: VID.

Chen, Yin-Zu. 2001. Interne Aspekte des Netzwerks und seine Handlungsfähigkeiten. Zeitschrift für Frauenforschung und Geschlechterforschung 19(1+2): 151-162.

Dautzenberg, Kristi, Doris Fay und Patricia Graf (Hrsg.). 2013. Aufstieg und Ausstieg. Wiesbaden: Springer VS. 
DeWelde, Kris und Sandra Laursen. 2011. The glass obstacle course: Informal and formal barriers for women PhD. students in STEM Fields. International Journal of Gender, Science and Technology 3(3): 572-595.

Dörre, Klaus und Matthias Neis. 2008. Forschendes Prekariat? S. 127-142 in Die Beschäftigungssituation von wissenschaftlichem Nachwuchs, hrsg. von Stephan Klecha und Wolfgang Krumbein. Wiesbaden: VS.

Durkheim, Emile. 1988. Über soziale Arbeitsteilung. Frankfurt a. M.: Surkamp.

Elg, Ulf und Karin Jonnergard. 2010. Included or excluded? Gender and Education 22(2): 209-225.

European Commission. 2013. She Figures 2012. Gender in Research and Innovation. Luxembourg: European Commission.

Ferguson, Kathy. 1984. The Feminist Case Against Bureaucracy. Philadelphia: Temple University Press.

Fieder, Martin, Susanne Huber, Fred Bookstein, Karoline Iber, Katrin Schäfer, Georg Winckler und Bernhard Wallner. 2005. Status and reproduction in humans: New evidence for the validity of evolutionary explanations on basis of a university sample. Ethology 111(10): 940-950.

Fritsch, Nina-Sophie. 2014. Warum Wissenschaftlerinnen die Universität verlassen. SWS-Rundschau 54(2): 159-180.

Froschauer, Ulrike. 2008. Organisationen. S. 186-201 in Forschungs- und Anwendungsbereiche der Soziologie, hrsg. von Rudolf Forster. Wien: Facultas Verlags- und Buchhandels AG.

Froschauer, Ulrike. 2012. Organisationen in Bewegung. Beiträge zur interpretativen Organisationsanalyse. Wien: Facultas.

Gardina, Maria, Marika Tiggemann, Hugh Kearns und Kelly Marshall. 2007. Show me the money! Higher Education Research \& Development 26(4): 425-442.

Gersik, Connie, Jean Bartunek und Jane Dutton. 2000. Learning from academia: The importance of relationships in professional life. Academy of Management Journal 43(6): 1026-1044.

Graf, Patricia, Judith Reißner und Sylvia Schmid. 2013. Wenn mein Team erfolgreich ist, bin ich es auch? S. 67-94 in Aufstieg oder Ausstieg, hrsg. von Kristi Dautzenberg, Doris Fay und Patricia Graf. Wiesbaden: Springer VS.

Heintz, Bettina und Eva Nadai. 1998. Geschlecht und Kontext. De-Institutionalisierungsprozesse und geschlechtliche Differenzierung. Zeitschrift für Soziologie 27(2): 75-93.

Hördt, Olga. 2006. Spitzenpositionen für Spitzenleistungen? Wiesbaden: Deutscher Universitätsverlag.

Kahlert, Heike. 2011. "Cooling out" und der riskante Weg an die Spitze. S. 105-124 in Forschung und Förderung, hrsg. von Jutta Wergen. Berlin: LIT-Verlag.

Kahlert, Heike. 2013. Riskante Karrieren. Opladen: Barbara Budrich.

Kanter, Rosabeth Moss. 1977. Men and Women of the Corporation. New York: Basic Books.

Kjeldal, Sue-Ellen, Jennifer Rindfleish und Alison Sheridan. 2005. Deal making and rule breaking: Behind the façade of equity in academia. Gender and Education 17(4): 431-447.

Klecha, Stephan und Melanie Reimer. 2008. Wissenschaft als besonderer Arbeitsmarkt. S. 13-87 in Die Beschäftigungssituation von wissenschaftlichem Nachwuchs, hrsg. von Klecha, Stephan und Wolfgang Krumbein. Wiesbaden: VS.

Krimmer, Holger, Freia Stallmann, Markus Behr und Annette Zimmer. 2003. Karrierewege von ProfessorInnen an Hochschulen in Deutschland. Münster: BMBF.

Lamnek, Siegfried. 1995. Qualitative Sozialforschung. München: Beltz.

Lind, Inken. 2007. Ursachen der Unterrepräsentanz von Wissenschaftlerinnen - Individuelle Entscheidungen oder strukturelle Barrieren? S. 59-86 in Exzellenz in Wissenschaft und Forschung, hrsg. von Wissenschaftsrat. Köln: BMBF.

McGuire, Gail und Jo Reger. 2003. Feminist co-mentoring: A model for academic professional development. NWSA Journal 15(1): 54-72. 
Merton, Robert. 1948. The self-fulfilling prophecy. Antioch Review 8(2): 193-210.

Müller, Ruth. 26.10. 2012. Life at the bottleneck. Career Magazine, http://sciencecareers.sciencemag. org/career_magazine/previous_issues/articles/2012_10_26/caredit.a1200120 (05.09.2014).

Nassehi, Armin. 1997. Inklusion, Exklusion-Integration, Desintegration. S. 113-148 in Was hält die Gesellschaft zusammen? hrsg. von Wilhelm Heitmeyer. Frankfurt a. M.: Campus.

Parsons, Talcott. 1975. Gesellschaften. Evolutionäre und komparative Perspektiven. Frankfurt a. M.: Suhrkamp.

Portes, Alejandro und Julia Sensenbrenner. 1993. Embeddedness and immigration. The American Journal of Sociology 98(6): 1320-1350.

Pringle, Rosemary. 1989. Secretaries Talk. London und New York: Verso Books.

Prskawetz, Alexia, Tomas Sobotka, Isabella Buber, Henriette Engelhardt, Richard Gisser. 2008. Persistent low fertility since the mid-1980s. Demographic Research 19(12): 293-360.

Raddon, Arwen. 2002. Mothers in the academy: Positioned and positioning within discourses of the "successful academic" and the "good mother". Studies in Higher Education 27(4): 387-403.

Rafnsdottir, Gudbjörg und Thamar Heijstra. 2013. Balancing work-family life in academia: The power of time. Gender, Work and Organization 20(3): 283-296.

Riegraf, Birgit. 1996. Geschlecht und Mikropolitik. Opladen: Leske+Budrich.

Riegraf, Birgit. 2013. Theoretische Erörterungen. S. 17-160 in Geschlecht und Organisation, hrsg. von Ursula Müller, Birgit Riegraf und Sylvia Wilz. Wiesbaden: VS.

Statistik Austria. 2011. Erhebung über Forschung und experimentelle Entwicklung 2011. Wien: Statistik Austria.

Strauss, Anselm und Juliet Corbin. 1996. Grounded Theory. Weinheim: Beltz.

uni:data. 2014. Datawarehouse Hochschulbereich. Wien: BMWFW.

Weber, Max. 1919. Wissenschaft als Beruf. München und Leipzig: Duncker\&Humboldt.

Wilz, Sylvia. 2002. Organisation und Geschlecht. Opladen: Leske+Budrich.

Wilz, Sylvia. 2013. Geschlechterdifferenzierung von und in Organisationen. S.150-160 in Geschlecht und Organisation, hrsg. von Ursula Müller, Birgit Riegraf, und Sylvia Wilz. Wiesbaden: VS.

Witzel, Andreas. 2000. Das problemzentrierte Interview. Forum Qualitative Sozialforschung 1(1), http:// nbn-resolving.de/urn:nbn:de:0114-fqs0001228, (11.03.2013).

Wolfinger, Nicholas, Mary Ann Mason und Marc Goulden. 2008. Problems in the pipeline: Gender, marriage, and fertility in the ivory tower. The Journal of Higher Education 79(4): 388-405.

Wroblewski, Angela und Andrea Leitner. 2011. Excellentia Evaluationsbericht. Wien: IHS. 